\title{
Surface Plasmon Electron Tomography viewed as an Inverse Hough Transform
}

\author{
G. Möbus, Z. Saghi, W. Guan \\ Dept. Engineering Materials, University of Sheffield, Mappin Street, Sheffield S1 3JD, UK
}

Surface plasmon EFTEM images (e.g. [1]) are a prominent example of the more general class of materials surface signals used for projection imaging. Rather than providing a projection relationship typically applicable for tomography, e.g. image intensity proportional to the atomic number density integrated along the ray of radiation, the signal is reversely related to the distance from the object surface. Examples of this class other than EFTEM comprise edge enhancing image post processing, or dark field techniques with a small transfer window along the thickness scale. All these properties require major modifications of standard tomography procedures and interpretations when applied to a tilt series for 3D object reconstruction. Fig. 1a illustrates the difference in projections expected from a surface signal of a nanoparticle, and a "bulk"-signal from the shell of a same-sized core-shell particle.

Morphology reconstruction of regularly shaped nanoparticles is possible by Geometric Tomography [2]:

(i) shape-from-silhouette was introduced into electron tomography to reconstruct heavy-atom and crystalline materials [3,4]: Using binarised images of any TEM imaging mode, i.e. the shadow, support or silhouette of a particle, and given a shape-constraint, which requires every cross-section perpendicular to the rotation axis to be convex ("axial convexity"), it can be shown that a full error-free surface reconstruction is possible.

(ii) shape-from-contour was introduced as a modification of shape-from-silhouette [3], where the projection images only show non-zero pixels at the edge of the projected object, without binarisation.

It has long been known that the Hough transform is intrinsically linked to the Radon transform as a special case, e.g. when dealing with objects or sceneries, in the simplest case, consisting of straight lines or edges [5]. The (forward) Hough transform returns angle, $\Theta$, and offset from origin, r, of a straight line $\mathrm{L} €\{\mathrm{x}, \mathrm{y}\}$ in Hough space as a peak $H €(r, \Theta)$, and optionally the line length via the peak intensity. Start and end point of the line are lost. The inverse operation, consequently, tracks the Hough-peak backwards into a line of infinite length. When applied to a test-object shaped as a rectangle (Fig 1b), the result will be a crossing-line pattern [3] enclosing, rather than reconstructing, the surface shape (Fig 1c). For roundish objects the excess lines outside the true object will smear into a low-intensity background, as sketched for one of the rounded corners in Fig 1c, and, after thresholding, an estimate of the true surface can be recovered. The inverse Hough transform is therefore a suitable description of the foundations of the shape-from-contour approach.

Applied to the problem of surface plasmon EFTEM images, the shape-from-contour technique has the promise to reconstruct surface morphology in spite of the dependency of signals on viewing angle: Fig 2 shows input images (one member of tilt series) of an Al nanoparticle cluster with surface signal (also catching some $\mathrm{C}$ ) around $7.5 \mathrm{eV}$ (slit $5 \mathrm{eV}$ ) in comparison with a bulk plasmon image for the $\mathrm{Al}$ core at $15 \mathrm{eV}$ and oxide+carbon shell at $23.5 \mathrm{eV}$ (slit $5 \mathrm{eV}$ ), along with profile plots. Fig 3 shows the central cross section through the backprojected tomogram, illustrating the tracking of the particle surfaces by short line segments. Prospective advantages are improved sharpness of the tomogram, elimination of artefacts typical to CT of crystalline materials in TEM, and the possibility of evaluation of localised plasmon spots. Disadvantages include the ambiguous results possible for objects with irregular concavities, and objects with a large variation in flat surface segment length. [6]

\section{References}

[1] W. Sigle, J. Nelayah, C.T. Koch, P.A. VanAken, Optics Letters 34 (2009), 2150-2152.

[2] R. J. Gardner, Geometric Tomography, Plenum Press, New York, 1995.

[3] Z.Saghi, X. Xu, G Möbus, J. Microscopy 232 (2008) 186-195.

[4] Z.Saghi et al., Appl.Phys.Lett. 93 (2008) 153102.

[5] N. Aggarwal, W.C. Karl, IEEE transact. im. proc. 15 (2006) 5827.

[6] This work was supported by EPSRC under grant number GR/S85689/01. 

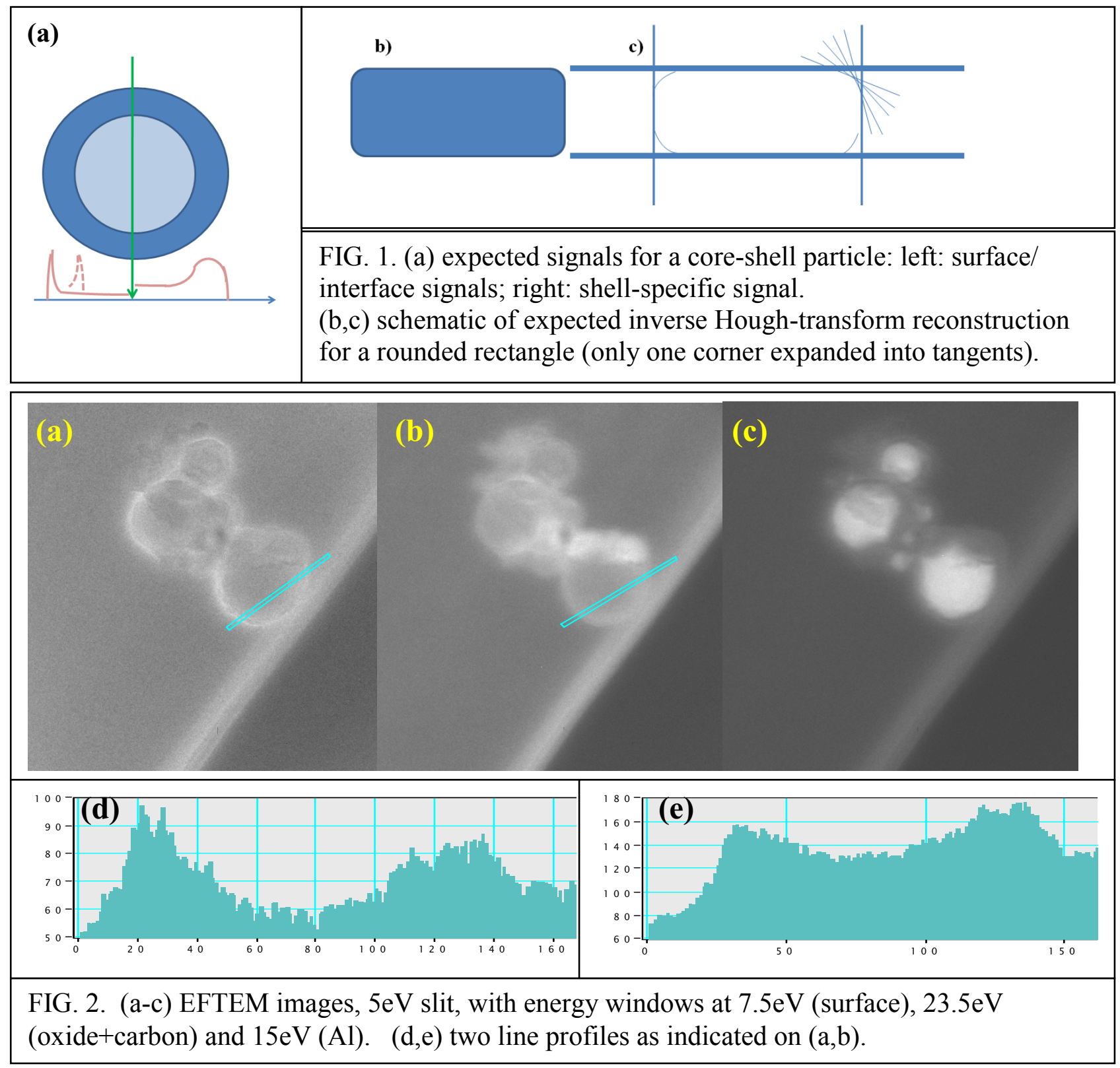

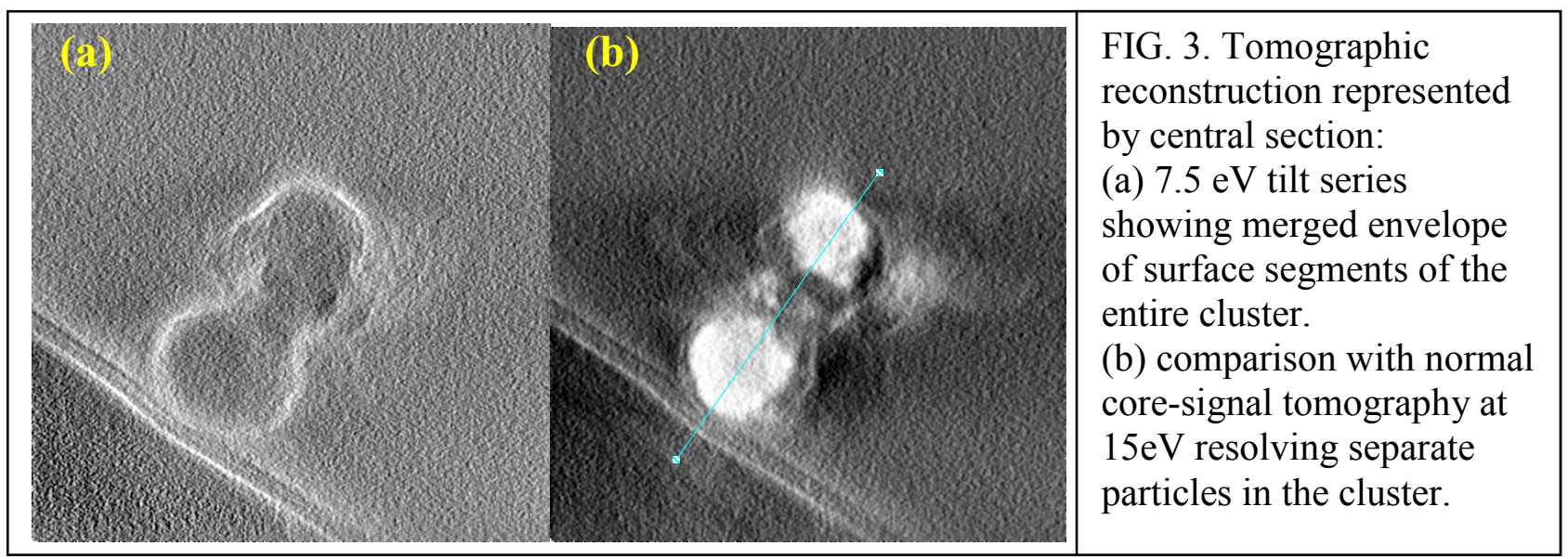

